\title{
Catgut Versus Polypropylene Sutures for Transcolumellar Incision Closure in Open Rhinoplasty: A Retrospective Cohort Study
}

\author{
Saud Aldhabaan ${ }^{1}$, Jibril Y. Hudise ${ }^{2}$, Mohammed ALqarny ${ }^{3}$, Ahmed Alarfaj ${ }^{2}$ \\ 1. Otolaryngology, King Faisal Medical City, Abha, SAU 2. Otolaryngology, King Abdulaziz University Hospital, Riyadh, \\ SAU 3. Otolaryngology, University of Bisah, Bisha, SAU
}

Corresponding author: Saud Aldhabaan, saldhabaan@ksu.edu.sa

\section{Abstract \\ Background}

Open rhinoplasty, including the transcolumellar approach, is commonly performed as it has fewer side effects and provides superior anatomical control to the surgeons compared to closed rhinoplasty. However, the postoperative scar outcomes, such as scar appearance, vary depending on the type of suture used in wound closure, and the optimal suture type is not firmly established.

\section{Objective}

To compare the impact of catgut versus polypropylene sutures on the postoperative transcolumellar scar outcomes and patient satisfaction following open rhinoplasty.

\section{Methods}

This retrospective cohort study, including 100 patients who underwent transcolumellar open rhinoplasty, was conducted at otolaryngology department of King Abdulaziz University Hospital, Riyadh, KSA. The patients were divided into two groups: the propylene suture group (group 1), which included 15 males and 35 females with a mean age of 31.5 years and underwent surgery using propylene sutures, and the catgut suture group (group 2), which included 10 males and 40 females with a mean age of 30.5 years and underwent surgery using catgut sutures. The postoperative transcolumellar scar outcomes, as determined by visual analogue scale (VAS) and Stony Brook Scar Evaluation Scale (SBSES) scores, and patient satisfaction, as assessed using a self-assessment scale, were compared between groups.

\section{Results}

The scars were unnoticeable in the majority of both groups: $88 \%$ in group 1 and $86 \%$ in group 2 . The VAS and SBSES scores did not significantly differ between groups. Patients' satisfaction rates were also comparable and did not significantly differ between groups $(\mathrm{p}=0.341)$.

Received 08/03/2020

Review began 08/03/2020 Review ended 08/05/2020 Published 08/15/2020

() Copyright 2020

Aldhabaan et al. This is an open access article distributed under the terms of the Creative Commons Attribution License CC-BY 4.0., which permits unrestricted use, distribution, and reproduction in any medium, provided the original author and source are credited.

\section{Conclusion}

Both catgut and polypropylene sutures lead to similar outcomes and patient satisfaction rates in terms of postoperative rhinoplasty transcolumellar scars. Thus, catgut may be the optimal suture for closing transcolumellar incisions following open rhinoplasty.

Categories: Otolaryngology, Plastic Surgery, Anatomy

Keywords: rhinoplasty, transcolumellar incision, catgut suture, polypropylene suture

\section{Introduction}

Rhinoplasty is a common surgical intervention aiming to preserve the aesthetic and functional properties of the nose. This surgery is also applied to reconstruct the deformed nose resulting from traumatic injuries, maxillary surgeries, or birth defects [1-3]. There are two main approaches to rhinoplasty: open and closed rhinoplasty. Open rhinoplasty is performed externally via an alar base or transcolumellar incision [4]. The transcolumellar scar provides superior anatomical and surgical control and generally heals well without complications using non-absorbable monofilament sutures, such as polypropylene [5]. However, follow-up visits are required to remove these sutures, leading to significant discomfort [6]. Absorbable sutures that can resorb spontaneously without affecting the aesthetics of the scar are a viable alternative [7,8]. Catgut is an absorbable, monofilament suture that consists of organic material derived from animal intestines, and can therefore elicit an allergic reaction and affect wound healing [9].

However, Sharma and Mehrotra reported that surgeons' fear of using catgut is unwarranted as it led to complications such as wound dehiscence and infection in only 15 (4.3\%) of 350 surgeries and provided 


\section{Cureus}

excellent scar healing in 322 (92\%) cases [10]. In contrast, polypropylene is a synthetic suture that causes a limited allergic reaction and does not adhere to the tissues. However, it can result in wound sinus formation, pain, palpable knots, and rate of wound infection can be up to $24 \%$ [11]. Previous studies investigating the use of absorbable and non-absorbable sutures in open rhinoplasty reported comparable results with both sutures. Thus, the optimal suture type has not been established [6-8].

An ideal suture is cheap, reliable, does not cut through the skin, provides superior handling and anatomical control during surgery, and promotes excellent wound healing post-surgery [12]. In the present study, we aimed to compare the impact of catgut and polypropylene sutures on postoperative scar outcomes and patients' satisfaction.

\section{Materials And Methods}

\section{Patients}

This retrospective cohort study was done to assess the postoperative wound scar outcomes and satisfaction rates with the use of catgut versus polypropylene sutures in patients who underwent tanscolumellar open rhinoplasty. We retrospectively looked into patient procedure records of open rhinoplasty using transcolumellar approach done by two surgeons and performed postoperative photography on the scars of the patients. The study was conducted at otolaryngology department of King Abdulaziz University Hospital, Riyadh, KSA between May 2019 and May 2020.

The study included patients who were 18 years or above and underwent open rhinoplasty for improving nose aesthetics with transcolumellar incision and the wound closure was done using catgut or polypropylene sutures. The exclusion criteria include patients below 18 years, patients who spent less than one year following their surgery, or had a rhinoplasty done secondary to trauma or congenital anomalies.

\section{Assessment}

The cases were followed-up after a year via telephone consultation, and self-assessment tools were used to assess the healing and satisfaction rates among the patients. A visual analogue scale (VAS) was used to categorize the postoperative scar outcome into unnoticeable, noticeable but acceptable, and noticeable and not acceptable. Assessment of patient photography (basal view) after one year of surgery, by two rhinoplastic surgeons (the same surgeons who did the surgeries) using Stony Brook Scar Evaluation Scale (SBSES) was carried out in order to categorize scar outcomes as poor (score 0-1), moderate (score 2-3), and good (score 4-5) [13]. Patient satisfaction was measured using a self-assessment scale ranging from 0 to 10 , where 0 indicated complete dissatisfaction and 10 indicated total satisfaction. Any disagreement among the assessors was resolved through mutual consultation and concurrence.

\section{Statistical analysis}

Statistical Package for the Social Sciences (SPSS), v 21.0 (IBM Corp., Armonk, NY) was used to analyze the data. The Chi-squared test was used to evaluate statistical significance, and a p-value of $\leqslant 0.05$ was considered significant.

\section{Results}

Our study included 100 participants who were divided into two groups. Group 1 included 50 participants who underwent transcolumellar incision surgery using interrupted polypropylene sutures. This group had a mean age of $31.4 \pm 6.5$ years and included 35 (70\%) females and 15 (30\%) males. Group 2 underwent transcolumellar incision surgery using catgut sutures. This group had a mean age of 30.5 6.3 years and included $40(80 \%)$ females and $10(20 \%)$ males (Table 1$)$.

\begin{tabular}{|c|c|c|}
\hline Variables & Group 1 (Polypropylene) & Group 2 (Catgut) \\
\hline Mean age (years) & $31.4 \pm 6.5$ & $30.5 \pm 6.3$ \\
\hline \multicolumn{3}{|l|}{ Sex } \\
\hline Females, n (\%) & 35 (70\%) & $40(80 \%)$ \\
\hline Males, $\mathrm{n}(\%)$ & $15(30 \%)$ & $10(20 \%)$ \\
\hline
\end{tabular}

TABLE 1: Patient demographics 


\section{Cureus}

We used the VAS to assess the patients' perception of their scars. In group 1, 44 (88\%), five (10\%), and one (2\%) patients reported having unnoticeable, noticeable but acceptable, and noticeable and unacceptable scars, respectively. In group 2, 43 (86\%) and seven (14\%) patients reported having unnoticeable and noticeable but acceptable scars, respectively. None of the participants in group 2 reported having noticeable and unacceptable scars. The differences between groups regarding patients' perception of the postoperative scar outcome were not significant $(\mathrm{p}=1.000)$ (Table 2).

\begin{tabular}{|c|c|c|c|c|}
\hline Group & Unnoticeable scars, n (\%) & Noticeable but acceptable, n (\%) & Noticeable and unacceptable, n (\%) & $\mathrm{p}$-value \\
\hline Group 1 & $44(88 \%)$ & $5(10 \%)$ & $1(2 \%)$ & \multirow{2}{*}{1.000} \\
\hline Group 2 & $43(86 \%)$ & $7(14 \%)$ & $0(0)$ & \\
\hline \multicolumn{5}{|c|}{ Stony Brook Scar Evaluation Scale (SBSES) analysis } \\
\hline & Poor score (0-1), n (\%) & Moderate score (2-3), n (\%) & Good score (4-5), n (\%) & $\mathrm{p}$-value \\
\hline Group 1 & $1(2 \%)$ & $6(12 \%)$ & $43(86 \%)$ & \multirow{2}{*}{0.221} \\
\hline Group 2 & $0(0 \%)$ & $5(10 \%)$ & $45(90 \%)$ & \\
\hline
\end{tabular}

\section{TABLE 2: VAS and SBSES analysis for both groups}

VAS, visual analogue scale; SBSES, Stony Brook Scar Evaluation Scale

Table 2 summarizes the SBSES scores. The majority of patients in both groups rated their scars outcomes as 'good' (scored 4 or 5) $(\mathrm{p}=0.225)$.

When asked to rate their satisfaction regarding scar outcomes on a scale of $0-10$, most of the participants in both groups reported satisfaction scores of $\geqslant 8$. The mean satisfaction scores were 9.0 and 9.2 for groups 1 and 2, respectively $(\mathrm{p}=0.341)$ (Table 3).

\begin{tabular}{|l|l|l|l|l|}
\hline Group & Lowest Rating & Highest Rating & Mean rating & Standard deviation \\
\hline Group 1 & 7.0 & 10.0 & 9.0800 & 0.82906 \\
\hline Group 2 & 7.0 & 10.0 & 9.2200 & 0.84007 \\
\hline
\end{tabular}

TABLE 3: Satisfaction scores from both groups

\section{Discussion}

The main purpose of the current study was to determine the impact of two different suture materials on aesthetic outcomes and patient satisfaction following open rhinoplasty. Our study showed that both suture types did not differ in terms of their aesthetic outcomes and patient satisfaction.

Ashraf et al. studied scar healing and patient satisfaction following transcolumellar septorhinoplasty. The sutures used in the study were non-absorbable Prolene ${ }^{\circledR}$ and absorbable Vicryl Rapide ${ }^{\circledR}$. The study did not report any statistically significant $(\mathrm{p}=0.39$ ) difference in scar rating and patient satisfaction with the use of either suture materials [14]. In another report, Alijanpour et al. analyzed 15 research papers comparing absorbable and non-absorbable sutures for outcomes including wound aesthetics and comfort in patients undergoing open rhinoplasty. The authors concluded that both suture types produced similar scar aesthetics. However, the patients reported greater satisfaction with the use of absorbable sutures because these sutures did not require removal [15]. This aspect has been discussed in several other studies wherein researchers did not notice any difference between aesthetic outcomes with the use of absorbable or nonabsorbable sutures in ear, nose, and throat or other facial surgeries [16-18]. Our current findings are consistent with the literature as we demonstrated that absorbable and non-absorbable sutures do not differ in terms of wound aesthetics and patient satisfaction scores.

To the best of our knowledge, our study is the first of its kind to use catgut as an absorbable suture in comparison to a non-absorbable suture (polypropylene) for assessing wound healing and patient 
satisfaction following transcolumellar open rhinoplasty. Surgeons are often concerned about the use of catgut due to the possibility of cross-sensitivity as it contains organic material, which might affect wound healing and aesthetics. However, this concern has not been clinically validated. Gazivado et al. compared wound healing and the incidence of complications such as wound infection and local reactions with the use of different types of absorbable sutures (catgut, Dexon, and Vicryl). The results suggested that none of the suture materials hindered wound healing, and there was a low incidence of wound complications with all of the used suture materials that did not reach statistical significance [19]. Other reports advocate the use of catgut as an absorbable suture as it is absorbable, has good tensile strength, is more comfortable for the patients as it spares them the pain of suture removal, leads to excellent wound healing, and has comparable rates of complications to other absorbable and non-absorbable suture materials $[14,15,19,20]$.

\section{Conclusions}

Our study has demonstrated that both absorbable catgut and non-absorbable polypropylene sutures can be used for the closure of transcolumellar incisions following open rhinoplasty. We found no significant difference in wound healing and patient satisfaction with the use of either of the suture materials. Therefore, catgut may be the optimal suture for closing transcolumellar incisions following open rhinoplasty as it gives comparable aesthetic outcomes to polypropylene and saves surgeons' time.

\section{Additional Information}

\section{Disclosures}

Human subjects: Consent was obtained by all participants in this study. Health Sciences Colleges Research on Human Subjects King Saud University College of Medicine issued approval E-19-3982. To: Prof. Ahmad Alarfaj Department of ENT King Abdul Aziz University Hospital Principal Investigator CC: Dr. Saud Abdulwahab Aldhabaan saldhabaan@ksu.edu.sa Dr. Jibirl Yahya Hudse Co - Investigators Subject: Approval of Research Project No. E-19-3982 Study title: Catgut Versus Polypropylene Sutures for Transcolumellar Incision Closure in Open Rhinoplasty: A Retrospective Cohort Study Dear Prof. Ahrnad Alarfaj, I am pleased to inform you that your above-mentioned research project submitted to the IRB was reviewed and approved on 16 May 2019 (11 Ramadan 1440). You are now granted permission to conduct this study given that your study does not disclose participant's identity and poses no risk to the patients. As principal Investigator, you are required to abide by the rules and regulations of the Kingdom of Saudi Arabia and the research policies and procedures of the KSU IRB. If you make any changes to the protocol during the period of this approval, you must submit a revised protocol to the IRB for approval prior to implementing the changes. Please quote the project number shown above in any future correspondence or follow-ups related to this study. This approval is for a period of one (1) year commencing from the date of this letter. If you wish to have your protocol approved for continuation, please submit a completed request for re-approval of an approved protocol form (KSU-IRB-017E) at least 30 days before the expiry date. Failure to receive approval for continuation before the expiration date will result in automatic suspension of the approval of this protocol on the expiration date. Information collected following suspension is unapproved research and can never be reported or published as research data. We wish you success in your research and request you to keep the IRB informed about the progress and final outcome of the study in a regular basis. If you have any question, please feel free to contact me. Thank you! Sincerely Yours, Prof. Abdulrahman Alsultan Chairman of IRB Health Sciences Colleges Research on Human Subjects King Saud University College of Medicine P. O. B ox 7605 Riyadh 11472 K.SA. Email: aalsultan@ksu.edu.sa . Animal subjects: All authors have confirmed that this study did not involve animal subjects or tissue. Conflicts of interest: In compliance with the ICMJE uniform disclosure form, all authors declare the following: Payment/services info: All authors have declared that no financial support was received from any organization for the submitted work. Financial relationships: All authors have declared that they have no financial relationships at present or within the previous three years with any organizations that might have an interest in the submitted work. Other relationships: All authors have declared that there are no other relationships or activities that could appear to have influenced the submitted work.

\section{References}

1. Raithatha R, Naini F, Patel S, et al.: Long-term stability of limiting nasal alar base width changes with a cinch suture following Le Fort I osteotomy with submental intubation. Int J Oral Max Surg. 2017, 46:13721379. 10.1016/j.ijom.2017.04.027

2. Rohrich R, Ahmad J: Rhinoplasty. Plast Reconstr Surg. 2011, 128:49e-73e. 10.1097/PRS.0b013e31821e7191

3. Tasman A: Rhinoplasty-indications and techniques. GMS Curr Top Otorhinolaryngol Head Neck Surg. 2007, 6:

4. Inanli S, Sari M, Yanik M: A new consideration of scar formation in open rhinoplasty . J Craniofac Surg. 2009, 20:1228-1230. 10.1097/scs.0b013e3181ace082

5. Dayan E, Rohrich R: Developing consistency in rhinoplasty. Plast Reconstr Surg Glob Open. 2020, 8:e2679. 10.1097/GOX.0000000000002679

6. Alinasab B, Haraldsson P: Rapid resorbable sutures are a favourable alternative to non-resorbable sutures in closing transcolumellar incision in rhinoplasty. Aesthetic Plast Surg. 2016, 40:449-452. 10.1007/s00266016-0649-2

7. Kundra RK, Newman S, Saithna A, et al.: Absorbable or non-absorbable sutures? A prospective, randomized evaluation of aesthetic outcomes in patients undergoing elective day-case hand and wrist surgery. Ann $\mathrm{R}$ 
Coll Surg Engl. 2010, 92:665-667. 10.1308/003588410x12699663905113

8. Al-Mubarak L, Al-Haddab M: Cutaneous wound closure materials: an overview and update. J Cutan Aesthet Surg. 2013, 6:178-188. 10.4103/0974-2077.123395

9. Engler R, Weber C, Turnicky R: Hypersensitivity to chromated catgut sutures: a case report and review of the literature. Ann Allergy. 1986, 56:317-320.

10. Sharma A, Mehrotra S: Is catgut really obsolete? Experience with buried subcuticular catgut sutures in operative wounds. Med J Armed Forces India. 2002, 58:217-220. 10.1016/s0377-1237(02)80133-2

11. Kiran Shankar H: A comparative study of outcome of the absorbable suture polydioxanone and nonabsorbable suture polypropylene in laparotomy wound closure. Int J Res Med Sci. 2016, 4:2084-2088. 10.18203/2320-6012.ijrms20161765

12. Islam A, Ehsan A: Comparison of suture material and technique of closure of subcutaneous fat and skin in caesarean section. N Am J Med Sci. 2011, 3:85-88. 10.4297/najms.2011.385

13. Nguyen T, Feldstein S, Shumaker P, et al.: A review of scar assessment scales . Semin Cutan Med Surg. 2015, 34:28-36. 10.12788/j.sder.2015.0125

14. Ashraf N, Foden N, D'souza A: Closure of the trans-columellar incision in open septorhinoplasty in 100 patients: use of an absorbable multifilament suture. Clin Otolaryngol. 2017, 42:1085-1087. 10.1111/coa.12861

15. Alijanpour S, Armoeyan M, Shahroudi P: Closure of the transcolumellar incision in open rhinoplasty: absorbable or nonabsorbable suture?. Biomed J Sci Technical Res. 2019, 21:15550-15552. 10.26717/bjstr.2019.21.003541

16. Gillanders SL, Anderson S, Mellon L, et al.: A systematic review and meta-analysis: do absorbable or nonabsorbable suture materials differ in cosmetic outcomes in patients requiring primary closure of facial wounds?. J Plast Reconstr Aes. 2018, 71:1682-1692. 10.1016/j.bjps.2018.08.027

17. Parell G, Becker G: Comparison of absorbable with nonabsorbable sutures in closure of facial skin wounds . Arch Facial Plast Surg. 2003, 5:488-490. 10.1001/archfaci.5.6.488

18. Kilavuz A, Bayram A, Serin G: Comparison of absorbable and nonabsorbable sutures in columellar incision closure in rhinoplasty and their effects to postoperative scar. Facial Plast Surg. 2017, 33:661-664. 10.1055/s0037-1608668

19. Gazivoda D, Pelemiš D, Vujašković G: A clinical study on the influence of suturing material on oral wound healing. Vojnosanit Pregl. 2015, 72:765-769. 10.2298/vsp140401064g

20. Kim H, Hwang K, Yun SM: Catgut and its use in plastic surgery. J Craniofac Surg. 2020, 31:876-878. $10.1097 /$ scs.0000000000006149 